\title{
Meniscal substitution, a developing and long-awaited demand
}

\author{
Philipp W. Winkler ${ }^{1,2}$, Benjamin B. Rothrauff ${ }^{1,3}$, Rafael A. Buerba' ${ }^{1}$ Neha Shah', Stefano Zaffagnini", \\ Peter Alexander ${ }^{3}$ and Volker Musahl $1^{*}$
}

\begin{abstract}
The menisci represent indispensable intraarticular components of a well-functioning knee joint. Sports activities, traumatic incidents, or simply degenerative conditions can cause meniscal injuries, which often require surgical intervention. Efforts in biomechanical and clinical research have led to the recommendation of a meniscus-preserving rather than a meniscusresecting treatment approach. Nevertheless, partial or even total meniscal resection is sometimes inevitable. In such circumstances, techniques of meniscal substitution are required. Autologous, allogenic, and artificial meniscal substitutes are available which have evolved in recent years. Basic anatomical and biomechanical knowledge, clinical application, radiological and clinical outcomes as well as future perspectives of meniscal substitutes are presented in this article. A comprehensive knowledge of the different approaches to meniscal substitution is required in order to integrate these evolving techniques in daily clinical practice to prevent the devastating effects of lost meniscal tissue.
\end{abstract}

Keywords: Knee, Meniscus, Allograft, Transplantation, Scaffold, Tissue engineering, Substitute

\section{Background}

The knee is the most commonly injured part of the body among young athletes [1], with isolated meniscal injuries occurring in approximately $15 \%$ of acute knee traumas associated with hemarthrosis [2]. The incidence of acute meniscal tears in combined ligamentous injuries is even higher, ranging up to $82 \%$ [3]. Differing in etiology and pathogenesis, degenerative meniscal injuries more often affect the elderly population and increase with age, with an estimated prevalence of over $50 \%$ between 70 and 90 years of age $[4,5]$.

Intensive research efforts have established a comprehensive knowledge of the menisci and their essential role for long-term knee function. This is reflected in the decreasing number of meniscal resections and the increasing number of meniscal repairs, highlighting a trend towards an evidence-based and meniscus-preserving approach in the treatment of meniscal tears. Among U.S.

\footnotetext{
* Correspondence: musahlv@upmc.edu

${ }^{1}$ Department of Orthopaedic Surgery, UPMC Freddie Fu Sports Medicine

Center, University of Pittsburgh, 3200 S. Water St, Pittsburgh, PA 15203, USA

Full list of author information is available at the end of the article
}

orthopedic surgeons, a decrease of $17 \%$ and an increase of $37 \%$ for meniscectomy and meniscal repair cases per surgeon, respectively, was observed from 2004 to 2012 [6]. Nevertheless, depending on the meniscal tear pattern, the tear size, location, quality of the meniscal tissue, and the stability of the meniscal tear and the knee joint, some meniscal tears are not reparable. In a recent study, $65 \%$ of 2066 meniscal tears were considered as irreparable [7]. Thus, a partial or total meniscectomy sometimes remains the best or only treatment option, although the devastating effects and radiographic changes of meniscectomy have been established [8]. Several studies have remarkably demonstrated the increased rate of knee osteoarthritis (OA), requirement for total knee arthroplasty, and the decreased clinical and functional outcomes after partial and total meniscectomy for up to 40 years follow-up [9-12]. Consequently, many efforts have been made to develop meniscal substitutes in order to prevent early-onset knee OA, a condition that typically results in a partial- or total knee replacement [13-15].
Springer Open

(c) The Author(s). 2020 Open Access This article is licensed under a Creative Commons Attribution 4.0 International License, which permits use, sharing, adaptation, distribution and reproduction in any medium or format, as long as you give appropriate credit to the original author(s) and the source, provide a link to the Creative Commons licence, and indicate if changes were made. The images or other third party material in this article are included in the article's Creative Commons licence, unless indicated otherwise in a credit line to the material. If material is not included in the article's Creative Commons licence and your intended use is not permitted by statutory regulation or exceeds the permitted use, you will need to obtain permission directly from the copyright holder. To view a copy of this licence, visit http://creativecommons.org/licenses/by/4.0/. 
The purpose of the present article is to provide a comprehensive review on the development, current state of research, clinical relevance, and future perspectives of meniscal substitutes.

\section{Anatomical and biomechanical considerations}

Due to the unique composition and three-dimensional structure, the menisci are indispensable for physiological knee function. Key functions of the menisci include load-bearing, improvement of the femoro-tibial congruency, and, in combination with the meniscal ligament complex, a secondarily stabilizing effect. Further important roles are the contribution to joint lubrication, proprioception, and the distribution of intraarticular nutrients [16]. Given the relative movement between the menisci and the tibial plateau, the menisci form a dynamically acting joint socket which enhances the femoro-tibial congruency across the entire range-ofmotion (ROM) $[17,18]$. This dynamic behavior is especially important when considering the fixation technique of meniscal allografts in order to best restore the native knee mechanics and kinematics [19].

There are three distinct layers when describing the cross-sectional area of the meniscal tissue [20]. Thick bundles of circumferentially orientated collagen fibers are located in the central main layer, which are responsible for the mechanical properties of the menisci, especially for tensile strength. Additional fibers are radially orientated, or build a meshwork in the superficial and lamellar layer, contributing to the anisotropic behavior of the meniscal tissue $[16,20]$.

The sophisticated combination of composition, morphology, and anatomical attachments of the menisci, transforms the axially acting compression force during load-bearing into a circumferentially orientated tensile force (hoop-stress) [16]. As a result, $50 \%$ and $70 \%$ of the compartmental load is absorbed by the medial and lateral meniscus, respectively [21]. Following a total medial meniscectomy, the local peak contact pressure in the medial compartment increases by approximately 235\%, highlighting the importance of the medial meniscus in load-bearing [22]. Similar results have been observed for the lateral compartment after total lateral meniscectomy [23]. Radial meniscal tears lead to a partial or total disruption of the circumferentially orientated collagen fibers. As a result, no hoop-stress can be transferred, which leads to pathological meniscal extrusion [24]. This condition causes an increase in the tibiofemoral contact pressure, which is why it is often termed "functional meniscectomy "[25-27]. Meniscal extrusion has gained interest in recent years and can be quantified by MRI or ultrasound [24, 28, 29]. It has been shown, that excessive meniscal extrusion is an indirect sign for radial tears as well as for meniscal root lesions [24, 29]. Radial meniscal displacement of $>3 \mathrm{~mm}$ is often considered as a cut-off value from physiologic to pathologic extrusion. However, a recent study has shown that meniscal extrusion can be a temporary and reversible observation after strenuous physical activity [28]. Therefore, the critical value for physiological meniscal extrusion continues to be the subject of current research.

The vascular supply of the menisci is limited to the peripheral $10-25 \%$ by a perimeniscal capillary plexus [30]. This refers to zone 1 according to the proposed radial zones of the menisci (Fig. 1) [3, 31]. The more central zones 2 and 3 have less vascular supply. Therefore, the healing potential of meniscal tears is limited, especially for tears located in zone 3.

\section{Knee after total meniscectomy}

Partial or total meniscectomy leads to an alteration of the biomechanics and kinematics of the knee [22, 32, 33]. Although meniscal resection may be followed by a rapid improvement of clinical symptoms, a subset of patients suffer from nagging pain after a certain period of time. This clinical presentation is termed "post-meniscectomy syndrome" and may be accompanied by radiological signs such as bone marrow edema [34]. Given the bony morphology of the tibiofemoral joint, it is important for the menisci to increase the tibiofemoral congruency. This is particularly true for the lateral compartment due to the convex lateral tibial plateau, which is reflected in the devastating effects following total or partial lateral meniscectomy [21, 35-38]. One study has shown that in patients undergoing total meniscectomy, the need for total knee arthroplasty is 132 times higher at 40-years follow-up due to symptomatic knee OA compared to an age-matched regional control population [11]. Non-surgical treatment for post-meniscectomy syndrome includes physical therapy, activity modification, and the use of non-steroidal antiinflammatory drugs [34]. However, the evidence for nonsurgical treatment remains limited and therefore surgical treatment options have to be considered, especially in young and active patients.

\section{Alignment and stability}

Lower limb alignment essentially affects the load distribution across the medial and lateral compartment. Increasing varus malalignment and increasing loss of medial meniscal tissue (i.e. partial/total medial meniscectomy) lead to elevated tibiofemoral contact pressure in the medial compartment [39, 40]. Furthermore, a positive correlation between medial meniscal extrusion and tibiofemoral contact pressure exists [41]. This provides evidence that the medial meniscus is subjected to increased stress in varus malalignment. As previously mentioned, the menisci act as secondary restraints to anterior tibial and combined rotatory loads, becoming 


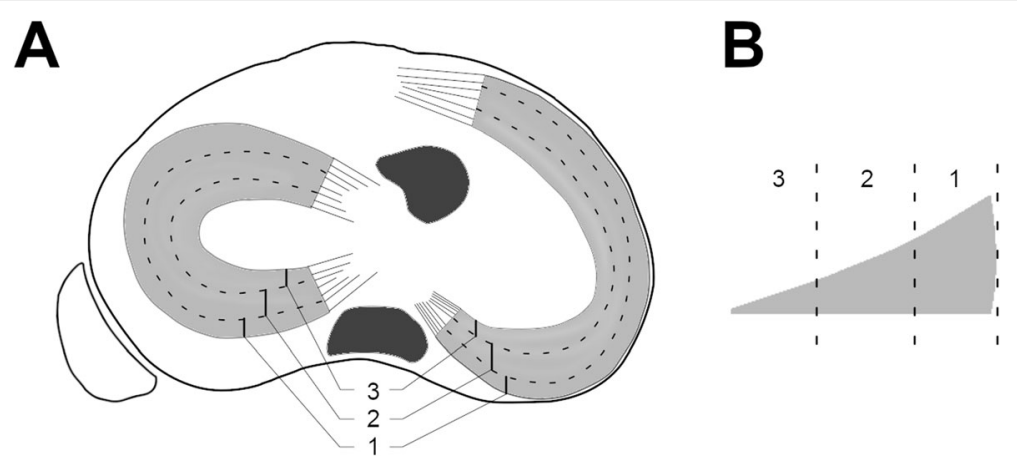

Fig. 1 Meniscal zones according to [3, 31]. a Proximal view of the tibial plateau. b Cross-sectional area of a representative meniscus. The dotted lines divide the menisci into three different zones: 1, peripheral zone; 2, middle zone; 3, central zone

primary stabilizers in the anterior cruciate ligament (ACL) deficient knee [32]. In addition, ACL deficiency exposes the menisci to increased movement, deformation and stress [42, 43]. Clinically, chronic knee instability in patients with ACL deficiency increases the risk of medial meniscus injuries. Even a delay of 6 months in ACL reconstruction significantly increases the odds of sustaining a secondary medial meniscal lesion, which almost doubles after 12 months [44, 45].

Taken together, it is recommended to assess and, if necessary, address lower limb alignment and knee instability prior to or in combination with meniscal substitution to protect the meniscal substitute.

\section{Principles of meniscal substitution}

Four main principles for the replacement of meniscal tissue exist. Total replacement can be performed with autogenous [46, 47] or allogenic transplants [48-51] as well as with artificial meniscus prostheses [52, 53]. For partial replacement, after extensive partial meniscectomy, artificial scaffold-based meniscal substitutes are available $[54,55]$.

Historically, total meniscal substitution was performed by using autografts like tendon, fat, or perichondral tissue. The autografts are intended to provide a threedimensional scaffold, which facilitates the immigration of endogenous cells to stimulate a remodeling process. As a result, meniscus-like tissue is formed [56-58]. Similarly, total meniscal replacement by meniscal allograft transplantation (MAT) uses a human donor meniscus, which also provides a scaffold while simultaneously best replicating the morphological characteristics and biomechanical properties of the lost meniscal tissue [59]. Total medial meniscal replacement can also be performed by the use of artificial meniscus prostheses. Non-degradable, anatomically or non-anatomically shaped meniscus prostheses are inserted into the medial compartment to replace the lost meniscal tissue $[52,53]$. To date, no evidence is available for lateral meniscal replacement by meniscus prostheses. Artificial scaffold-based meniscal substitutes, designed for partial meniscal replacement, provide an organically compounded three-dimensional scaffold which facilitates the ingrowth of cells to form new meniscus-like tissue. However, the initially implanted substitute is not designed to restore the biomechanical properties of the lost meniscal tissue. The ingrowth of fibrochondrocyte-like cells induces tissue formation and remodeling, which then resembles the biomechanical properties of the native meniscus [55, $60,61]$.

\section{Autologous substitutes}

Various types of autologous tissue for meniscal substitution have been reported. In one study, total medial meniscal replacement was performed with an ipsilateral mid-third patellar tendon autograft in a sheep model. After 12 months, tissue remodeling of the tendon autograft (tendon-meniscus) with incorporated chondrocytelike cells could be observed. Despite morphological and histological similarities of the native meniscus and the tendon-meniscus, the biomechanical properties of the autograft were significantly worse compared to the native meniscus [58]. Similar morphological results but worse biomechanical properties were found when using a pediculated infrapatellar fat pad autograft in another sheep model [57]. A further attempt for total meniscal replacement was the use of autologous perichondral tissue harvested from the lower rib in a sheep model. Again, the autograft underwent remodeling but possessed inferior biomechanical properties compared to the native meniscus. Interestingly, the integrity of the articular surface was better preserved in the perichondralmeniscus group than in the meniscectomized group [56]. In spite of some promising results in animal studies, no favorable clinical results have been reported for autologous meniscal substitutes and therefore no routine clinical use has been established $[46,47]$. 


\section{Allogenic substitutes \\ Meniscal allograft transplantation (MAT)}

Meniscal allograft transplantation is the standard-of-care for the treatment of symptomatic post-meniscectomy syndrome after sub-/total meniscal resection. Indications and contraindications for MAT are listed in Table 2.

After a thorough arthroscopic debridement of the affected compartment, a size-matched meniscal allograft is inserted into the knee through one of the arthroscopic portals or a mini-open arthrotomy. In principle there are two different techniques for intraarticular graft fixation: with bone block and suture-only. For the bone block fixation technique, a distinction between medial and lateral MAT has to be made. For lateral MAT in the bone block technique, a tibial trough, which connects the anterior and posterior meniscal roots, is created. For pressfit fixation, the bone block, which is attached to the meniscal allograft roots, is pushed into the trough [19]. In contrast, for medial MAT, two small bone plugs, which are attached to the anterior and posterior root of the medial meniscal allograft, are inserted into the respective tibial bone tunnel and fixed in a transtibial pullout fashion [62]. Additional soft tissue fixation is performed utilizing inside-out, outside-in or all-inside meniscal sutures, as appropriate. For the suture-only fixation technique (Fig. 2), the meniscal allograft roots are fixed by two transtibial pull-out sutures. Additional fixation of the meniscal rim is performed by the use of inside-out, outside-in or all-inside meniscal sutures, as appropriate [19, 48-50, 59]. For rehabilitation, many different approaches are described after MAT. Mostly, ROM exercises are started within the first postoperative week to achieve full ROM after 8 weeks. With regard to weight-bearing, 4 weeks and 6 weeks are recommended to start partial and full weight-bearing, respectively [64].

As MAT has substantially evolved since the 1980s, clinical data with emphasis on the different fixation techniques, medial vs. lateral MAT, return-to-play, failure and re-operation rates as well as long-term outcomes of many trials have been published. Overall, patient reported outcomes (PROs) improve significantly after MAT from preto postoperative, both for medial and lateral MAT as well as for the bone block and the suture-only fixation technique $[48-50,59]$. However, the superiority of either of the two fixation techniques remains unknown. In a recent matched-pair analysis, no difference between the bone block $(n=37)$ and the suture-only $(n=45)$ fixation technique could be observed regarding PROs and meniscal allograft extrusion measured by MRI [49]. In contrast, more favorable results are described for lateral MAT than for medial MAT. One meta-analysis comparing medial vs. lateral MAT showed that lateral MAT leads to higher Lysholm scores and improved pain relief compared to medial MAT at mid-term (5-10 years) and long-term (> 10 years) follow-up (FU) [65]. Encouraging clinical outcomes after MAT are also reflected in the high return-toplay rate. A recent systematic review reported a return-toplay rate of $77 \%(483 / 624)$ after MAT. The average reported time from surgery to return-to-play is 9 months and close to $70 \%(326 / 475)$ return to the same or a higher sports level [64]. However, the main goal of MAT is pain relief and a subsequent improved quality of life. This has to be discussed with every patient preoperatively to avoid unrealistic expectations. Accordingly, patients should be counseled against a return to high level sports. Although the weighted average satisfaction rate after MAT is $82 \%$, the respective complication rate is $11 \%$ [59]. One study demonstrated a re-operation rate of $32 \%$ after MAT (172 patients, 59 months mean FU) [51]. Tearing of the meniscal allograft is described to be the most common complication [59] and a simple arthroscopic debridement the most frequently performed re-operation procedure [51]. However, the survival rate of MAT is reported to be $86 \%$ (medial) / 89\% (lateral) at mid-term (5-10 years) and $52.6 \%$ (medial) / 57\% (lateral) at long-term (> 10 years) [65].

Taken together, MAT represents the standard-of-care in the treatment of patients with symptomatic postmeniscectomy syndrome. Yet this procedure has some inherent limitations. First, the meniscal allograft has to fit properly in size in order to achieve its desired benefits. Oversizing of the meniscal allograft results in increased tibiofemoral contact pressure compared to the native condition, since the oversized graft is not capable of transforming compressive load into hoop-stress and thus limits the ability of load transmission [66]. On the other hand, undersizing increases the stress within the meniscus, leading to a higher risk of graft failure [66]. Second, meniscal allografts are not readily available in many countries, which limits their broad clinical use.

\section{Artificial substitutes Scaffolds}

Two artificial, scaffold-based, and biocompatible meniscal substitutes for partial meniscal replacement are commercially available for clinical use. The collagen meniscus implant (CMI; Stryker Corporation, Kalamazoo, MI, USA), which consists of type-I collagen fibers derived from bovine Achilles tendons, has gained attention since the first clinical trial was published in 1997 [54]. The second, more recently developed artificial meniscal substitute is labelled Actifit ${ }^{\circ}$ (Orteq Sports Medicine Ltd., London, United Kingdom), which represents a synthetic hybrid of polycaprolactone (80\%) and polyurethane (20\%) and was first described in a clinical trial in 2011 [55]. A detailed synopsis of published clinical studies on 

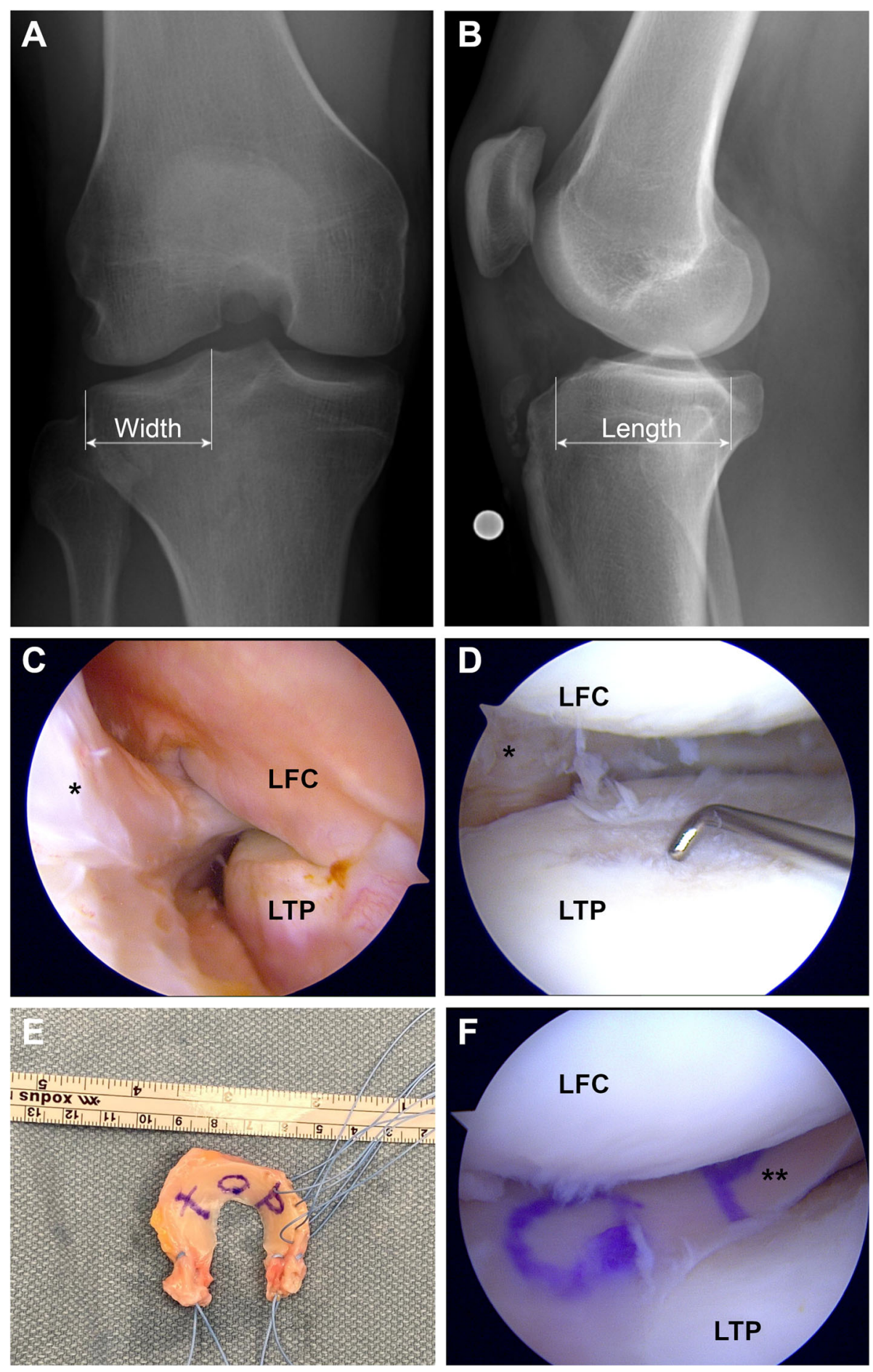

Fig. 2 Lateral meniscal allograft transplantation (MAT) by suture-only fixation technique in a patient having undergone subtotal lateral meniscectomy without rim preservation (right knee). Preoperative a) anterior-posterior (AP) and $\mathbf{b}$ ) lateral radiographs for sizing of the meniscal allograft. The width is measured on the AP radiograph as the distance from the lateral border of the tibial plateau to the apex of the lateral tubercle of the intercondylar eminence. The length is determined as $70 \%$ of the measured length of the lateral tibial plateau [63]. c) Arthroscopic view of the lateral gutter. Note the absence of meniscal tissue between the lateral femoral condyle (LFC) and the lateral tibial plateau (LTP); d) Arthroscopic view of the lateral compartment with grade 2 femoral and tibial cartilage lesions; e) Lateral meniscal allograft after preparation and application of the fixation sutures; $\mathbf{f}$ ) Arthroscopic view of the lateral compartment after lateral MAT; *,Remnant of the native lateral meniscus; ${ }^{* *}$, Lateral meniscal allograft in situ 
scaffold-based meniscal substitutes is shown in Table 1.

Once the indication (Table 2) for partial meniscal substitution has been proven and a thorough debridement of the partially meniscectomized area has been performed, the size of the scaffold is determined by an arthroscopic ruler. Thereafter, the implantation of the slightly oversized $(+10 \%$ of the debrided area) scaffold is performed arthroscopically. All-inside, inside-out, or outside-in suture techniques are used for substitute fixation to the surrounding meniscal tissue, as appropriate $[55,61,92]$. A schematic illustration and clinical examples of scaffold-based partial meniscal substitution are shown in Fig. 3 and Fig. 4, respectively. For postoperative rehabilitation, a hinged knee brace is recommended. Continuous passive ROM exercises limited to $60^{\circ}$ and $90^{\circ}$ of knee flexion for 3 to 6 weeks postoperatively, respectively, should be performed. Partial weight-bearing in full knee extension is allowed after 3 weeks, followed by free active ROM and full weight-bearing after 6 to 9 weeks $[55,61]$.

Almost every clinical trial investigating clinical outcomes after scaffold-based partial meniscal replacement reports improvements of PROs at follow up (Table 1). However, comparative studies are scarce [61, 78, 93]. A randomized, controlled, multicenter clinical trial showed that patients treated with CMI regained significantly higher activity (Tegner Activity Scale) and were more satisfied compared to patients treated with partial medial meniscectomy. No difference could be observed between the group with irreparable medial meniscal tears and acute replacement with CMI and the group treated with partial medial meniscectomy [61]. Similar results were shown by a randomized controlled trial with a minimum of 10 years follow up [93]. A recently published multicenter study showed an improvement in PROs after Actifit implantation at 2- and 5-years FU compared to the preoperative condition. Clinical outcome data are reported for 137 patients and show consistency between 2 and 5 years postoperatively. No difference could be demonstrated between medial and lateral implantation with a mean overall survival rate of the meniscal scaffold of $92 \%$ and $88 \%$ at 2 - and 5-years FU, respectively [67]. However, clinical failure, defined as scaffold-related infections, mechanical failure, chronic synovitis or the need for re-operation is reported in up to $8 \%$ and $32 \%$ for the CMI and Actifit ${ }^{\circ}$, respectively [67, 70, 71, 83, 87].

Since knee joint instability and lower limb malalignment are contraindications for scaffold-based partial meniscal substitution, concurrent interventions are frequently performed, with ACL reconstruction, high tibial osteotomy, and cartilage procedures being the most common $[67,72,77,78,89]$. A recent study showed that a combined high tibial valgus osteotomy and medial meniscus scaffold-based partial substitution does not lead to better clinical outcomes - based on PROs - than a combined high tibial osteotomy with partial medial meniscus resection. Therefore, the authors did not recommend the combination of high tibial valgus osteotomy and partial medial meniscal substitution, as lower limb realignment appeared to achieve the most important clinical improvement [78].

The clinical benefit of scaffold-based partial meniscal substitution is more evident in the chronic rather than acute settings (Table 1). This appears to be controversial, since a loss of meniscal tissue has been shown to instantly alter knee joint biomechanics [32, 33]. The rapid improvement of clinical symptoms after partial meniscectomy following an acute meniscal lesion may be an explanation. In contrast, the process of remodeling and adjustment to an implanted meniscal scaffold requires more time, but might be favorable regarding long-term outcomes [94], since tissue ingrowth within the scaffold and formation of meniscus-like tissue is evident according to histological analysis and second-look arthroscopy [54, 55, 61, 77, 88, 91, 92]. Consequently, the devastating effects of partial meniscectomy may be prevented and the development of knee OA delayed [9, 10, 12].

\section{Meniscus prostheses}

Anatomically [53, 95] and non-anatomically [52, 96] shaped artificial meniscal substitutes (prostheses) made of polycarbonate-urethane represent novel techniques for total meniscal replacement. In spite of intensive research efforts, experiences to date are limited.

In one study, the condition of an anatomically shaped total meniscal substitute and its chondroprotective effect was investigated with 12 months $\mathrm{FU}$ in a goat model. Although the integrity of the total meniscal substitute, in terms of wear and deformation, was maintained, no difference in the cartilage histopathological degeneration could be observed between the groups of total meniscal substitution, meniscal allograft transplantation and total meniscectomy. Additionally, the total meniscal substitute was not chondroprotective compared to a non-operative control group [53]. A biomechanical study on human cadaveric specimens showed that the same anatomically shaped total meniscal substitute could not restore native contact mechanics and knee kinematics. Based on the results of this study, there is no significant difference between total meniscal substitution and MAT [95].

NUsurface ${ }^{\bullet}$ Meniscus Implant (Active Implants LLC, Memphis, TN, USA) represents a non-anatomically discoid-shaped, free floating and non-anchored meniscal substitute designed for total replacement of the medial meniscus $[52,96]$. A biomechanical study 
Table 1 Synopsis of clinical studies on scaffold-based meniscal substitutes (chronological order)

\begin{tabular}{|c|c|c|c|c|c|c|c|c|c|c|c|c|}
\hline Author & Year & $\mathrm{N}$ & Agel & $\begin{array}{l}01 \\
0 / 1 \\
0\end{array}$ & $\mathrm{FU}$ & Scaffold & $M / L$ & $A / C$ & Size ${ }^{\|}$ & $\begin{array}{l}\text { Assoc. } \\
\text { Procedures }\end{array}$ & Radiologic Outcomes & Clinical Outcomes \\
\hline & & & [years] & & [months] & & & & {$[\mathrm{mm}]$} & [\%] & & \\
\hline Toanen [67] & 2020 & 155 & 34 & $\begin{array}{l}109 / \\
46\end{array}$ & 60 & Actifit $^{\oplus}$ & $\begin{array}{l}101 / \\
54\end{array}$ & $14 / 141$ & 39.4 & $\begin{array}{l}\operatorname{HTO}(28), \\
\operatorname{ACLR}(19), C S \\
\text { (4) }\end{array}$ & $\begin{array}{l}\text { Genovese score, } \\
\text { extrusion, ICRS }\end{array}$ & $\begin{array}{l}\text { VAS, LS, IKDC, } \\
\text { KOOS, survival rate }\end{array}$ \\
\hline Schenk [68] & 2019 & 39 & 34 & $30 / 9$ & 36 & $\mathrm{CMl}$ & $32 / 7$ & $25 / 14$ & 48 & ACLR (62) & $\begin{array}{l}\text { Genovese score, } \\
\text { extrusion }\end{array}$ & $\begin{array}{l}\text { VAS, LS, IKDC, } \\
\text { Tegner, clinical } \\
\text { failure }\end{array}$ \\
\hline Monllau [69] & 2018 & 32 & 41 & $25 / 7$ & 70.8 & Actifit $^{\oplus}$ & $\begin{array}{l}21 / \\
11\end{array}$ & N/A & 40.9 & $\begin{array}{l}\text { ACLR (28), } \\
\text { HTO (41), PCLR } \\
\text { (3), CS (44) }\end{array}$ & $\begin{array}{l}\text { Genovese score, } \\
\text { extrusion, volume }\end{array}$ & $\begin{array}{l}\text { LS, IKDC, KOOS, } \\
\text { Tegner, }\end{array}$ \\
\hline Leroy [70] & 2017 & 15 & 30 & $8 / 7$ & 72 & Actifit $^{\oplus}$ & $6 / 9$ & $0 / 15$ & N/A & $\begin{array}{l}\text { ACLR (33), CS } \\
\text { (7) }\end{array}$ & $\begin{array}{l}\text { Genovese score, } \\
\text { extrusion, ICRS }\end{array}$ & $\begin{array}{l}\text { VAS, IKDC, KOOS, } \\
\text { failure rate }\end{array}$ \\
\hline $\begin{array}{l}\text { Dhollander } \\
\text { [71] }\end{array}$ & 2016 & 44 & 32 & $\begin{array}{l}24 / \\
20\end{array}$ & 60 & Actifit $^{\oplus}$ & $\begin{array}{l}29 / \\
15\end{array}$ & $4 / 40$ & 45.5 & $\begin{array}{l}\text { ACLR (9), HTO } \\
\text { (9) }\end{array}$ & $\begin{array}{l}\text { Genovese score, } \\
\text { extrusion, ICRS }\end{array}$ & $\begin{array}{l}\text { VAS, IKDC, KOOS, } \\
\text { survival rate }\end{array}$ \\
\hline Filardo [72] & 2016 & 16 & 45 & $9 / 7$ & 72 & Actifit $^{\oplus}$ & $12 / 4$ & N/A & N/A & $\begin{array}{l}\text { ACLR (38), } \\
\text { HTO (13), ME } \\
\text { (94), CS (13), O } \\
\text { (6) }\end{array}$ & $\begin{array}{l}\text { Genovese score, } \\
\text { extrusion }\end{array}$ & IKDC, Tegner \\
\hline $\begin{array}{l}\text { Schüttler } \\
\text { [73] }\end{array}$ & 2015 & 18 & 33 & N/A & 48 & Actifit $^{\oplus}$ & $18 / 0$ & $0 / 18$ & 44.5 & None & $\begin{array}{l}\text { Genovese score, } \\
\text { extrusion }\end{array}$ & $\begin{array}{l}\text { VAS, KOOS, KSS, } \\
\text { UCLA }\end{array}$ \\
\hline Faivre [74] & 2015 & 20 & 29 & N/A & 24 & Actifit $^{\oplus}$ & $8 / 12$ & $0 / 20$ & N/A & $\begin{array}{l}\text { Ligament } \\
\text { reconstruction } \\
\text { (20), CS (15) }\end{array}$ & $\begin{array}{l}\text { Signal intensity, } \\
\text { extrusion, cartilage } \\
\text { coverage index }\end{array}$ & IKDC, KOOS \\
\hline $\begin{array}{l}\text { Martín- } \\
\text { Hernández } \\
{[75]}\end{array}$ & 2015 & 10 & 31 & $4 / 6$ & 24 & Actifit $^{\oplus}$ & $9 / 1$ & $0 / 10$ & N/A & None & $\begin{array}{l}\text { Genovese score, } \\
\text { extrusion }\end{array}$ & VAS, LS, KOOS \\
\hline $\begin{array}{l}\text { Schüttler } \\
\text { [76] }\end{array}$ & 2015 & 18 & 33 & N/A & 24 & Actifit $^{\oplus}$ & $18 / 0$ & $0 / 18$ & 40.9 & None & $\begin{array}{l}\text { Genovese score, } \\
\text { extrusion }\end{array}$ & $\begin{array}{l}\text { VAS, KOOS, KSS, } \\
\text { UCLA }\end{array}$ \\
\hline Baynat [77] & 2014 & 18 & $20-46$ & $13 / 5$ & 24 & Actifit $^{\oplus}$ & $13 / 5$ & $0 / 18$ & N/A & $\begin{array}{l}\text { ACL (56), HTO } \\
\text { (56) }\end{array}$ & $\begin{array}{l}\text { Genovese score, } \\
\text { extrusion }\end{array}$ & LS, Histology \\
\hline Gelber [78] & 2014 & 30 & 51 & $\begin{array}{l}40 / \\
20\end{array}$ & 31.2 & Actifit $^{\oplus}$ & $60 / 0$ & $0 / 60$ & 40.3 & HTO (100) & $\begin{array}{l}\text { Radiographic evaluation } \\
\text { of limb alignment and } \\
\text { tibial slope }\end{array}$ & $\begin{array}{l}\text { VAS, IKDC, Kujala, } \\
\text { WOMET, }\end{array}$ \\
\hline $\begin{array}{l}\text { Bouyarmane } \\
\text { [79] }\end{array}$ & 2014 & 54 & 28 & $\begin{array}{l}37 / \\
17\end{array}$ & 24 & Actifit $^{\oplus}$ & $0 / 54$ & $0 / 54$ & 43 & $\begin{array}{l}\text { ACLR (17), } \\
\text { DFO (9) }\end{array}$ & N/A & VAS, IKDC, KOOS \\
\hline Kon [80] & 2014 & 18 & 45 & $11 / 7$ & 24 & Actifit $^{\oplus}$ & $13 / 5$ & $1 / 17$ & N/A & $\begin{array}{l}\text { ACLR (17), } \\
\text { Osteotomy } \\
\text { (22), CS (39), O } \\
(17)\end{array}$ & Genovese score & IKDC, Tegner \\
\hline $\begin{array}{l}\text { De Coninck } \\
\text { [81] }\end{array}$ & 2013 & 26 & 35 & $\begin{array}{l}12 / \\
12\end{array}$ & 24 & Actifit $^{\oplus}$ & $18 / 8$ & N/A & 43.2 & $\begin{array}{l}\text { ACLR (23), } \\
\text { HTO (4), DFO } \\
\text { (4), CS (4), } \\
\text { MAT (4) }\end{array}$ & $\begin{array}{l}\text { Meniscal rim thickness, } \\
\text { extrusion }\end{array}$ & $\begin{array}{l}\text { VAS, LS, IKDC, } \\
\text { KOOS }\end{array}$ \\
\hline $\begin{array}{l}\text { Bulgheroni } \\
\text { [82] }\end{array}$ & 2013 & 20 & 33 & $17 / 2$ & $24-46$ & Actifit $^{\oplus}$ & $17 / 3$ & $0 / 20$ & $\begin{array}{l}43 / \\
37^{* *}\end{array}$ & $\begin{array}{l}\text { ACLR (47), } \\
\text { HTO (37), DFO } \\
\text { (5) }\end{array}$ & Genovese score & $\begin{array}{l}\text { VAS, LS, Tegner, } \\
\text { second-look }\end{array}$ \\
\hline $\begin{array}{l}\text { Hirschmann } \\
\text { [83] }\end{array}$ & 2013 & 67 & 36 & $\begin{array}{l}47 / \\
20\end{array}$ & 12 & $\mathrm{CMl}$ & $\begin{array}{l}55 / \\
12\end{array}$ & $42 / 25$ & N/A & $\begin{array}{l}\operatorname{ACLR}(67), \\
\text { HTO (7), CS (4) }\end{array}$ & $\begin{array}{l}\text { Genovese score, } \\
\text { extrusion }\end{array}$ & $\begin{array}{l}\text { VAS, LS, IKDC, } \\
\text { Tegner, clinical } \\
\text { failure }\end{array}$ \\
\hline $\begin{array}{l}\text { Zaffagnini } \\
\text { [84] }\end{array}$ & 2012 & 24 & 36 & $20 / 4$ & 26 & $\mathrm{CMI}$ & $0 / 24$ & $7 / 17$ & 45.2 & $\begin{array}{l}\text { ACLR (17), CS } \\
(25), O(4)\end{array}$ & $\begin{array}{l}\text { Genovese score, Yulish } \\
\text { score }\end{array}$ & $\begin{array}{l}\text { VAS, LS, IKDC, } \\
\text { Tegner, EQ-5D }\end{array}$ \\
\hline Efe [85] & 2012 & 10 & 29 & $8 / 2$ & 12 & Actifit $^{\oplus}$ & $10 / 0$ & $0 / 10$ & 39.2 & None & $\begin{array}{l}\text { Genovese score, bone } \\
\text { bruise, ICRS, remaining } \\
\text { meniscus }\end{array}$ & $\begin{array}{l}\text { VAS, KOOS, KSS, } \\
\text { UCLA }\end{array}$ \\
\hline Verdonk [86] & 2012 & 52 & 31 & $\begin{array}{l}39 / \\
13\end{array}$ & 24 & Actifit $^{\oplus}$ & $\begin{array}{l}34 / \\
18\end{array}$ & $\begin{array}{l}2 / 46 \text { (4 } \\
\text { unknown) }\end{array}$ & 47.1 & $\operatorname{ACLR}(4)$ & ICRS & $\begin{array}{l}\text { VAS, LS, IKDC, } \\
\text { KOOS, SAE }\end{array}$ \\
\hline
\end{tabular}


Table 1 Synopsis of clinical studies on scaffold-based meniscal substitutes (chronological order) (Continued)

\begin{tabular}{|c|c|c|c|c|c|c|c|c|c|c|c|c|}
\hline Author & Year & $\mathrm{N}$ & Agel & $\begin{array}{l}0 / 1 \\
01 \\
0\end{array}$ & FU & Scaffold & $M / L$ & $\mathrm{~A} / \mathrm{C}$ & Size & $\begin{array}{l}\text { Assoc. } \\
\text { Procedures }\end{array}$ & Radiologic Outcomes & Clinical Outcomes \\
\hline Verdonk [55] & 2011 & 52 & 31 & $\begin{array}{l}39 / \\
13\end{array}$ & 12 & Actifit $^{\circledast}$ & $\begin{array}{l}34 / \\
18\end{array}$ & $\begin{array}{l}2 / 46 \text { (4 } \\
\text { unknown) }\end{array}$ & 47.1 & N/A & $\begin{array}{l}\text { Tissue ingrowth (DCE- } \\
\text { MRI), ICRS }\end{array}$ & $\begin{array}{l}\text { Pain, functionality, } \\
\text { quality of life, } \\
\text { Histology }\end{array}$ \\
\hline Zaffagnini & 2011 & 17 & 40 & $33 / 0$ & 133 & $\mathrm{CMl}$ & $33 / 0$ & $17 / 16$ & 36 & $\operatorname{ACLR}(12)$ & $\begin{array}{l}\text { Genovese score, Yulish } \\
\text { score, Radiographic } \\
\text { evaluation }\end{array}$ & $\begin{array}{l}\text { VAS, LS, IKDC, } \\
\text { Tegner, SF-36 }\end{array}$ \\
\hline Monllau [87] & 2011 & 25 & 29 & $20 / 5$ & 133.2 & $\mathrm{CMl}$ & $25 / 0$ & $20 / 5$ & 48.2 & $\begin{array}{l}\mathrm{ACLR}(56), \mathrm{CS} \\
\text { (4) }\end{array}$ & $\begin{array}{l}\text { Genovese score, } \\
\text { radiographic } \\
\text { progression of OA }\end{array}$ & VAS, LS, failure rate, \\
\hline $\begin{array}{l}\text { Bulgheroni } \\
\text { [88] }\end{array}$ & 2010 & 34 & 39 & $25 / 9$ & 60 & $\mathrm{CMl}$ & $34 / 0$ & $6 / 28$ & 45 & $\begin{array}{l}\text { ACLR (32), } \\
\text { HTO (6), CS (3) }\end{array}$ & $\begin{array}{l}\text { Genovese score, Yulish } \\
\text { score, radiographic } \\
\text { progression of OA }\end{array}$ & $\begin{array}{l}\text { LS, Tegner, } \\
\text { Histology }\end{array}$ \\
\hline Rodkey [61] & 2008 & 160 & 39 & $\begin{array}{l}243 / \\
68\end{array}$ & 59 & $\mathrm{CMl}$ & $\begin{array}{l}311 / \\
0\end{array}$ & $157 / 154$ & N/A & $\operatorname{ACLR}(27)$ & N/A & $\begin{array}{l}\text { VAS, LS, Tegner, } \\
\text { Histology }\end{array}$ \\
\hline Linke [89] & 2007 & 23 & 42 & N/A & 24 & $\mathrm{CMI}$ & $23 / 0$ & N/A & N/A & HTO (100) & N/A & VAS, LS, IKDC \\
\hline $\begin{array}{l}\text { Genovese } \\
{[90]}\end{array}$ & 2007 & 40 & 41 & $\begin{array}{l}27 / \\
13\end{array}$ & $12-24$ & $\mathrm{CMl}$ & $40 / 0$ & $28 / 12$ & N/A & $\begin{array}{l}\operatorname{ACLR}(40), \\
\operatorname{HTO}(3), C S(4)\end{array}$ & Genovese score & N/A \\
\hline $\begin{array}{l}\text { Steadman } \\
\text { [91] }\end{array}$ & 2005 & 8 & 40 & $8 / 0$ & 69.6 & $\mathrm{CMl}$ & $8 / 0$ & $1 / 8$ & N/A & None & $\begin{array}{l}\text { Signal intensity, } \\
\text { interface, cartilage, bone } \\
\text { marrow, radiographic } \\
\text { evaluation of OA }\end{array}$ & $\begin{array}{l}\text { VAS, LS, IKDC, } \\
\text { Tegner, Histology }\end{array}$ \\
\hline Rodkey [92] & 1999 & 8 & 40 & N/A & 24 & $\mathrm{CMI}$ & $8 / 0$ & $1 / 7$ & 42.5 & None & $\begin{array}{l}\text { Morphology, size, } \\
\text { radiographic } \\
\text { progression of OA }\end{array}$ & $\begin{array}{l}\text { VAS, LS, Tegner, } \\
\text { Histology }\end{array}$ \\
\hline Stone [54] & 1997 & 10 & 39 & $8 / 2$ & 36 & CMI & $10 / 0$ & $4 / 6$ & N/A & ACLR (20) & $\begin{array}{l}\text { Signal intensity, } \\
\text { interface, radiographic } \\
\text { evaluation of } O A\end{array}$ & $\begin{array}{l}\text { VAS, activity + } \\
\text { performance score, } \\
\text { overall knee rating, } \\
\text { Histology }\end{array}$ \\
\hline
\end{tabular}

Abbreviations: $A / C$ Acute/chronic; $A C L R$ Anterior cruciate ligament reconstruction; CMI Collagen meniscus implant; CS Cartilage surgery; DCE-MRI Dynamic contrastenhanced magnetic resonance imaging; DFO Distal femoral osteotomy; EQ-5D EuroQuol 5 dimensions; FU Follow-up; HTO High tibial osteotomy; ICRS International cartilage repair society; IKDC International knee documentation committee; KOOS Knee injury and osteoarthritis outcome score; KSS Knee society score; LS Lysholm score; $M / L$, medial/lateral; MAT Meniscal allograft transplantation; ME Meniscectomy; $N$ Number of patients treated with scaffold-based meniscal substitutes; $N / A$ Not available; O Others; OA Osteoarthritis; PCLR Posterior cruciate ligament reconstruction; SAE Scaffold-related serious adverse events; SF-36 Short form 36; UCLA University of California, Los Angeles activity scale; VAS Visual analogue scale; WOMET Western Ontario meniscal evaluation tool; ',mean age at surgery; ", mean defect size

Table 2 Indications and contraindications for scaffold-based meniscal substitutes and meniscal allograft transplantation

\begin{tabular}{|c|c|c|}
\hline & Scaffold-based meniscal substitution & Meniscal allograft transplantation \\
\hline Indications & $\begin{array}{l}\text { Clinical symptoms } \\
\text { s/p extensive partial meniscal resection } \\
\text { Stable meniscal rim } \\
\text { Intact meniscal roots } \\
\text { Chronic partial meniscal defect }\end{array}$ & $\begin{array}{l}\text { Clinical symptoms } \\
\text { s/p sub-/total meniscal resection } \\
\text { Insufficient meniscal rim } \\
\text { Insufficient meniscal roots }\end{array}$ \\
\hline Contraindications & $\begin{array}{l}\text { Age: > } 50 \text { years } \\
\text { BMl: }>35^{\mathrm{a}} \\
\text { Insufficient meniscal rim } \\
\text { Insufficient meniscal roots } \\
\text { Knee instability } \\
\text { Limb malalignment } \\
\text { Allergies to animal derived products } \\
\text { Meniscal defect limited to zone } 3 \text { (Fig. 1) } \\
\text { ICRS grade }>3 \\
\text { Active infection } \\
\text { Autoimmune diseases } \\
\text { Inflammatory arthritis }_{\text {Smoker }}{ }^{\mathrm{a}}\end{array}$ & $\begin{array}{l}\text { Age: > } 50 \text { years } \\
\text { BMI: }>35^{\mathrm{a}} \\
\text { Outerbridge grade III, IV } \\
\text { Fairbank grade }>2 \\
\text { Joint space narrowing } \\
\text { Knee instability } \\
\text { Limb malalignment } \\
\text { Active infection } \\
\text { Autoimmune diseases } \\
\text { Inflammatory arthritis }_{\text {Smoker }^{\mathrm{a}}}\end{array}$ \\
\hline
\end{tabular}




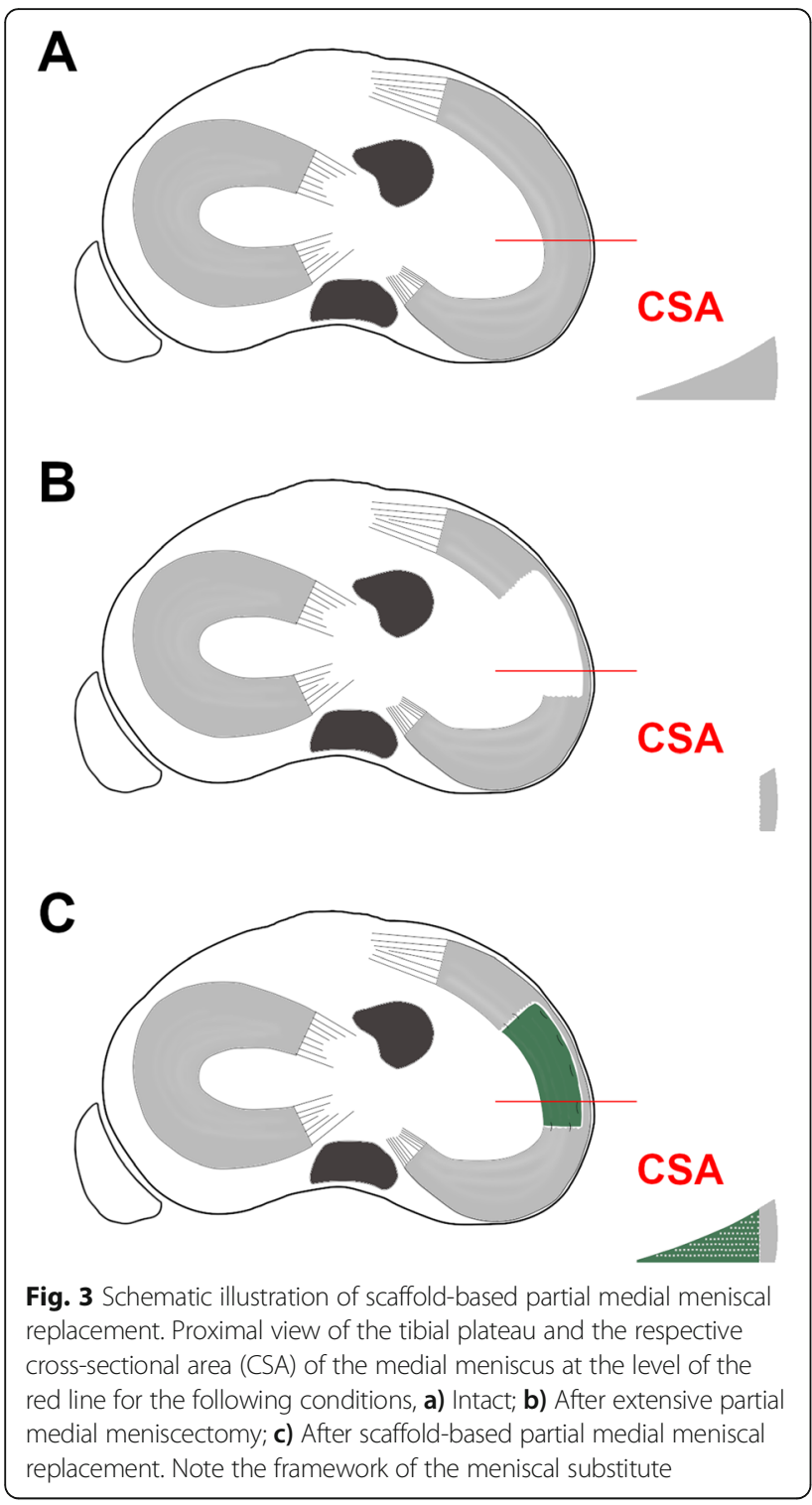

showed that implantation of the NUsurface ${ }^{\circ}$ Meniscus Implant restores the average and peak tibiofemoral contact pressure to $93 \%$ and $92 \%$, respectively, compared to the native medial meniscus [96]. In a caseseries of three prospectively enrolled patients, no differences for knee and meniscal kinematics between knees with implanted medial NUsurface ${ }^{\circ}$ Meniscus Implant and the contralateral healthy knees could be observed based on MRI studies. However, clinical outcome measures are not mentioned in this study [52]. Although the clinical use of the NUsurface ${ }^{\circ} \mathrm{Me}-$ niscus Implant started in Europe and Israel in 2008 and 2011, respectively, evidence-based clinical data remains largely absent. Two clinical trials are currently ongoing in the USA, with FDA approval for the NUsurface ${ }^{\bullet}$ Meniscus Implant still pending [97].

\section{Clinical indication}

Generally, the complaints and clinical symptoms of the patient are the primary predictors in treatment decisionmaking [98]. The ideal patient for scaffold-based meniscal substitution has a history of extensive partial meniscectomy with the peripheral meniscal rim remaining intact, pain localized to the respective joint line, full range of motion, has no signs of knee instability, no malalignment, or high-grade chondral defects, and has failed non-operative treatment $[61,67,93]$. On the other hand, an insufficient meniscal rim and a previously performed sub-/total meniscectomy represent contraindications for partial meniscal substitution and MAT is the standard-of-care in such cases [59]. Due to poor clinical data, autologous meniscal substitutes have not gained acceptance for clinical practice. Total meniscal replacement on the basis of meniscus prostheses has recently been established for clinical use. However, comprehensive clinical data are still pending.

The indications and contraindications for scaffold-based meniscal substitution and MAT are listed in Table 2.

\section{Tissue engineering and future perspectives}

Tissue engineering, under the broader umbrella of regenerative medicine, entails the independent or combined application of cells, bioactive agents (e.g., growth factors, viral vectors, small bioactive peptides), scaffolds, and biophysical stimuli, to fabricate biological substitutes that restore, maintain, or improve tissue function [99]. As noted above, clinically available meniscal substitutes, including non-meniscus autografts (e.g., tendon, adipose), MAT, meniscal prostheses, and scaffolds (i.e., CMI and Actifit ${ }^{\circ}$ ), each possess unique advantages and disadvantages. None restore the exact morphology, biochemical composition, ultrastructure, and cellular phenotypes of the native meniscus, let alone matching these properties for each unique patient. Meniscal tissue engineering ultimately seeks to overcome the limitations of current meniscal substitutes by fabricating a scaffold that is either seeded with cells prior to implantation or sequentially recruits and orchestrates meniscus-specific differentiation of endogenous progenitor cells, thereby serving as a patient-specific autograft.

Decellularized extracellular matrix (dECM) derived from allograft or xenogeneic meniscus largely possesses the ultrastructural, biochemical composition, and bioactive motifs of native meniscus with elimination of the immunogenic foreign cells $[100,101]$. The preserved microenvironment of the native tissue promotes meniscus- and even region-specific cellular differentiation by $\mathrm{dECM}[102,103]$. Preservation of bioactive motifs is possible with further processing of the meniscus dECM into powders, hydrogels, and water-soluble protein cocktails, which may impart meniscus-specific bioactivity when 

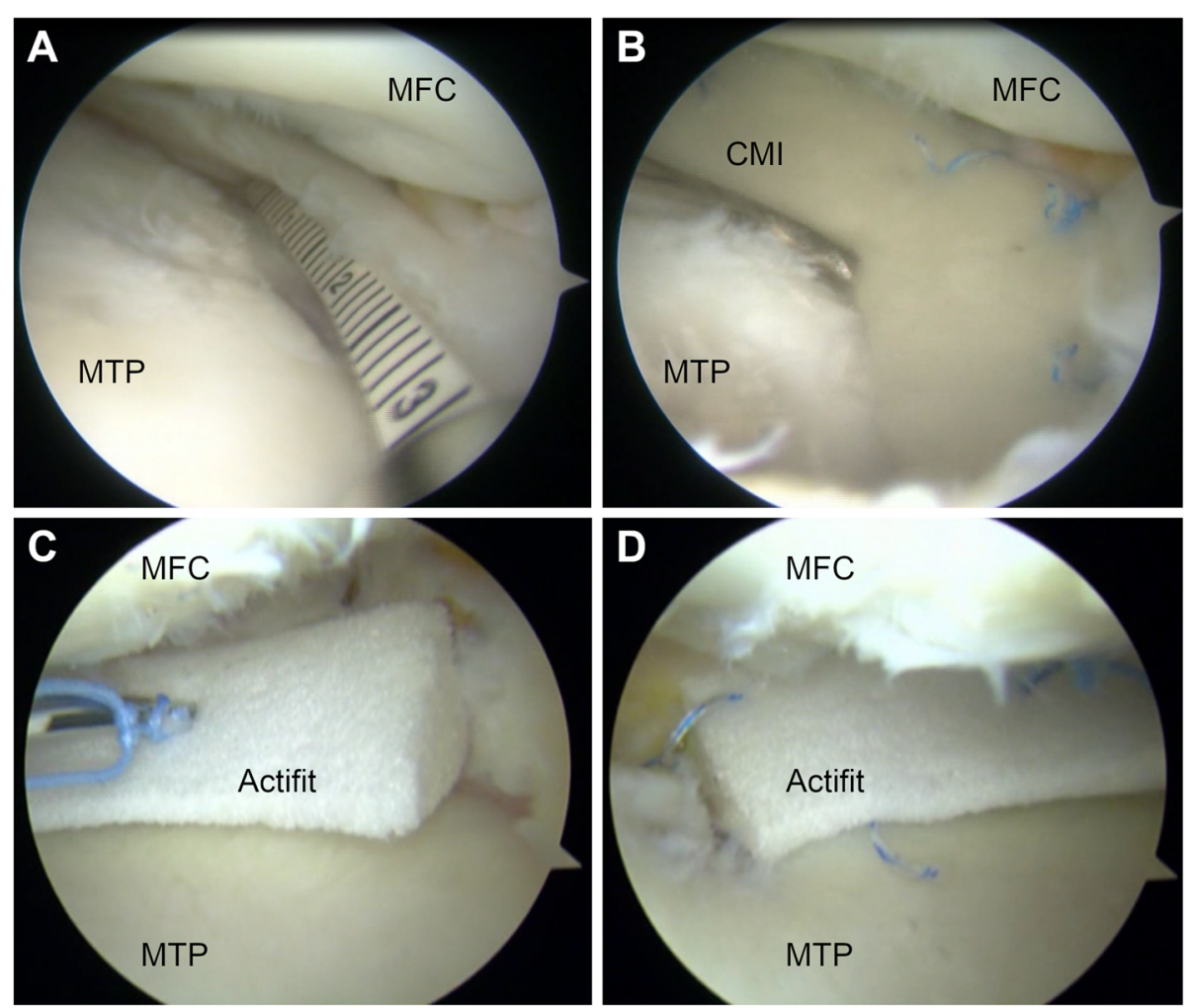

Fig. 4 Arthroscopic images of scaffold-based partial meniscal substitution. a) and b) Substitution of partial medial meniscus defect using the collagen meniscus implant (CMl; Stryker Corporation, Kalamazoo, MI, USA). c) and d) Substitution of partial medial meniscus defect using Actifit ${ }^{\oplus}$ (Orteq Sports Medicine Ltd., London, United Kingdom). a) Measurement of the defect size. b) CMI in situ. c) All-inside suture fixation of Actifit ${ }^{\circledR}$. d) Actifit ${ }^{\oplus}$ in situ. MFC, medial femoral condyle; MTP, medial tibial plateau. (Acknowledgement to Stefano Zaffagnini, who provided the images)

combined with other biomaterials [102, 104]. For instance, a 3-dimensional- (3D-) printed polycaprolactone (PCL) scaffold in the shape of the meniscus was augmented with meniscus dECM hydrogel [105]. When seeded with meniscal fibrochondrocytes (MFCs), the hybrid-scaffolds enhanced cell proliferation, chondrogenic differentiation, glycosaminoglycan and collagen production, and mechanical properties, as compared to the PCL scaffold without dECM [105]. Six months following implantation as a meniscal replacement into a rabbit model of total medial meniscectomy, the PCLdECM-MFC construct had similar histological structures, biochemical contents, and biomechanical properties as the native meniscus, a result that was superior to both a PCL scaffold and PLC-dECM construct [105].

While meniscus $\mathrm{dECM}$ has shown promising results in both in vitro and preclinical models, its procurement is labor-intensive and prone to batch-to-batch variability, with additional limitations in size matching for total meniscal replacement. The combined use of defined biodegradable polymers and bioactive factors (e.g., growth factors) affords greater control over the fabrication process of engineered scaffolds. Comprehensive reviews of the myriad materials and methods employed to fabricate tissue-engineered scaffolds have been published $[106,107]$. As an example, a PCL scaffold was 3Dprinted through computer-assisted design (CAD) modeling of a 3D laser scan of the native meniscus [108]. Using growth-factor impregnated microspheres of biodegradable polymers, the controlled-release of transforming growth factor beta 3 (TGF- $\beta 3$ ) and connective tissue growth factor (CTGF) was respectively localized to the inner and outer regions of the scaffold, providing region-specific chondrogenic and fibrochondrogenic differentiation of seeded mesenchymal stem cells (MSCs), as tested in vitro [108]. When implanted in an ovine model of medial meniscal replacement, the acellular scaffold with zone-specific TGF- $\beta 3$ (inner) and CTGF (outer) microspheres recruited endogenous progenitor cells, promoted type II collagen deposition in the inner region and type I collagen deposition in the outer region that was reminiscent of the native meniscus, and restored inhomogeneous mechanical properties [108]. These promising results were seen at 3 months following implantation. Unfortunately, when animals were followed up to 1 year, there was cartilage degeneration observed grossly and meniscal extrusion evident on MRI in most animals, suggesting that the engineered scaffold 
did not perfectly restore native meniscal function [109]. As for all tissue-engineered scaffolds, long-term studies examining the potential chondroprotective effect of the biomaterial are needed but infrequently performed. As with existing meniscal substitutes, controlling degradation/remodeling rates and preventing meniscal extrusion of engineered scaffolds remain challenges for optimizing the benefit of these promising biomaterials.

Meniscal replacement is the most ambitious application of tissue engineering, but the strategies may also be applied to promote localized meniscus regeneration, as for instance, to augment suture repair techniques. Intraarticular injection of MSCs into animal models of meniscus tears and/or partial meniscectomy have been found to improve healing and promote regeneration as determined by macroscopic observation, histological scoring, and MRI imaging [110-112]. More commonly, MSCs (or fibrochondrocytes/chondrocytes) seeded in a cell carrier (e.g., hydrogel, scaffold) are localized to the site of damage. Not only does the cell carrier concentrate the MSCs and their pro-healing paracrine factors at the tear site, but it can serve to concurrently release bioactive factors and/or provide structural support to the cells and resulting neotissue. In two related studies, injection of MSCs encapsulated in a thermosensitive [113] or photocrosslinkable [114] hydrogel into a meniscal tear in a rabbit or goat repair model, respectively, enhanced healing especially when the hydrogels where concomitantly supplemented with pro-chondrogenic TGF- $\beta$ isoforms. In the study using a goat model, the MSCs were rapidly isolated by enzymatic digestion of the infrapatellar fat pad, permitting a point-of-care (i.e., single stage) cell-based therapy for augmented meniscus repair [114] (Fig. 5).

Emerging technologies, including biomaterials, gene editing, cellular engineering, advanced imaging, and additive manufacturing (e.g. 3D printing), will provide expanding opportunity to repair, regenerate, or replace the injured meniscus. However, very few clinical studies have been performed at present. In the only prospective, randomized clinical study on MSC therapy for meniscus regeneration, 55 patients at 7 institutions who underwent partial medial meniscectomy were injected with one of three treatments - Group A: $50 \times 106$ allogeneic MSCs, Group 2: $150 \times 106$ allogeneic MSCs, or Group 3 (controls): sodium hyaluronate [115]. As determined by quantitative MRI, there was a significant increase in meniscal volume (defined a priori as $15 \%$ total meniscal volume) in $24 \%$ of patients in Group A, $6 \%$ of patients in Group B, and no patient in Group C. For those with osteoarthritic changes, both MSC groups reduced pain, as determined by a visual analog scale [115]. Several related case reports and case series with small sample sizes have been reported with generally positive findings, but
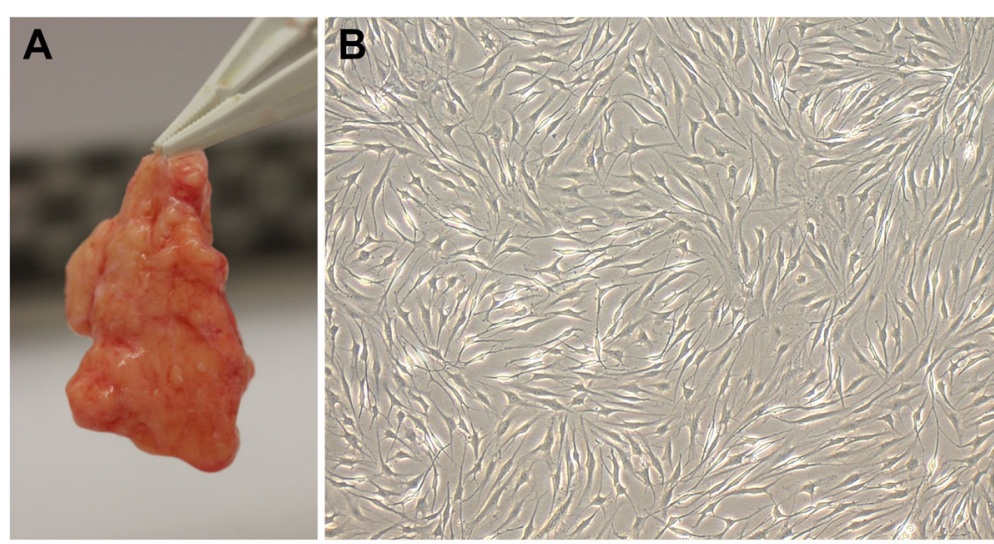

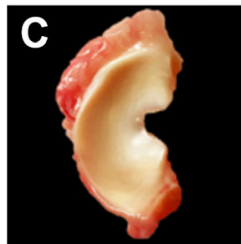

Control

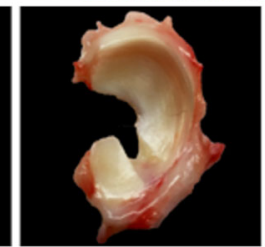

Untreated

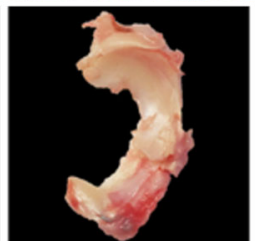

Repair

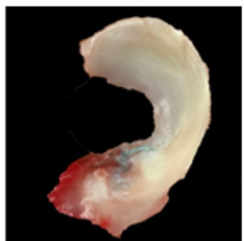

Augmented

Fig. 5 Augmented meniscal repair in a goat model. a) Isolated infrapatellar fat pad (IPFP) b) IPFP-derived mesenchymal stromal cells under microscope demonstrating characteristic spindle-shaped morphology c) Goat meniscus 6 months after creation of a critical-sized radial meniscal tear in 4 different groups: Control, no meniscal tear; Untreated, radial meniscal tear left in situ; Repair, radial tear repair with single horizontal mattress suture; Augmented, radial tear repair with single horizontal mattress suture + augmentation with cell-seeded hydrogel at the tear site. Suture repair minimized gap formation and neotissue formation was further improved with cell-based augmentation 
absent the inclusion of comparative groups (preferably placebo controls), conclusions on the putative clinical benefit of tissue engineering strategies addressing meniscus injury remain limited [116].

Implementation of meniscal tissue engineering strategies into clinical practice must address both technical and regulatory challenges. Understanding the mechanisms by which cell-based therapies, and tissue engineering constructs more broadly, exert their effect has been obscured in part by imprecise nomenclature and the inconsistent and insufficient reporting of details of both the biological product and the resulting outcome measures [117]. Several professional societies with interest in advancing cell-based therapies and tissue engineering have issued guidelines to address these past limitations [118-120]. Further limiting more rigorous clinical investigation on the topic is the regulatory landscape set forth by governing health agencies. Notably, the United States' Federal Drug Agency (FDA), which oversees the testing, approval, and use of cell and tissue products, categorizes nearly all tissue engineering constructs as "human cells, tissues, and cellular and tissue-based products (HCT/ Ps)", which require formal clinical trials to evaluate safety and efficacy [121]. Regulation is unequivocally needed to fulfill the FDA's mission of protecting public health, but the current regulation of tissue engineering products, including adults MSCs, creates financial barriers that have heretofore often been prohibitive for researchers, companies, and investors, to pursue more widespread clinical testing. Recognizing the promise in tissue engineering, the FDA developed a comprehensive regenerative medicine policy framework in 2017, as regulatory bodies increasingly engage clinicians, researchers, and companies, to spur innovation while ensuring safety and efficacy. Engagement of all parties will be needed to bring transformative technologies to fruition, thereby improving the care of patients with challenging meniscus injuries.

\section{Conclusion}

Remarkable research efforts over the past decades have enhanced the knowledge about the importance of the menisci for the knee, which has led to the development of meniscus-preserving and meniscus-replacing treatment options. While autologous meniscal substitution has not been successful, the techniques of meniscal allograft transplantation evolved to become the current standard-of-care for total meniscal insufficiency. Increasing clinical evidence for the efficacy of artificial scaffoldbased meniscal substitutes has emerged for the treatment of irreparable partial meniscal injuries. The feasibility of cell-seeded scaffolds, the preservation of the meniscal microenvironment to promote cell proliferation and differentiation, and the development of materials that mimic the mechanical and viscoelastic properties of the native menisci are owed to the progress in tissue engineering. Therefore, the future of meniscal substitution is encouraging with the goal of ultimately improving outcome for patients with post-meniscectomy syndrome.

\section{Abbreviations \\ 3D: 3-Dimensional; ACL: Anterior cruciate ligament; CMI: Collagen meniscus implant; CSA: Cross-sectional area; CTGF: Connective tissue growth factor; dECM: Decellularized extracellular matrix; FDA: Federal drug agency; FU: Follow-up; HCT/Ps: Human cells, tissues, and cellular and tissue-based products; MAT: Meniscal allograft transplantation; MFCs: Meniscal fibrochondrocytes; MSCs: Mesenchymal stem cells; OA: Osteoarthritis; PCL: Polycaprolactone; PROs: Patient reported outcomes; ROM: Range-of- motion; TGF- $\beta 3$ : Transforming growth factor beta 3}

\section{Acknowledgements}

Not applicable.

\section{Authors' contributions}

PWW, BBR, RBS and NS performed the literature review and primary manuscript preparation. VM, AP and SZ assisted with literature review, initial drafting of the manuscript, as well as editing and final draft preparation. All authors read and approved the final manuscript.

\section{Funding}

No funding was received for the preparation of this manuscript.

Availability of data and materials

Not applicable.

Ethics approval and consent to participate

Not applicable.

Consent for publication

Not applicable.

\section{Competing interests}

V.M. is a consultant for Smith \& Nephew and receives royalties from Springer Nature. S.Z. is a consultant for Depuy Synthes and Smith \& Nephew.

\section{Author details}

${ }^{1}$ Department of Orthopaedic Surgery, UPMC Freddie Fu Sports Medicine Center, University of Pittsburgh, 3200 S. Water St, Pittsburgh, PA 15203, USA. ${ }^{2}$ Department for Orthopaedic Sports Medicine, Klinikum rechts der Isar, Technical University of Munich, Ismaninger Str. 22, 81675 Munich, Germany. ${ }^{3}$ Center for Cellular and Molecular Engineering, University of Pittsburgh, 450 Technology Drive, Suite 239, Pittsburgh, PA 15219, USA. ${ }^{4} 2^{\circ}$ Clinica Ortopedica e Traumatologica, Istituto Ortopedico Rizzoli, IRCCS, University of Bologna, Bologna, Italy.

Received: 16 June 2020 Accepted: 2 July 2020

Published online: 25 July 2020

References

1. Darrow CJ, Collins CL, Yard EE, Comstock RD (2009) Epidemiology of severe injuries among United States high school athletes: 2005-2007. Am J Sports Med 37:1798-1805

2. DeHaven KE (1980) Diagnosis of acute knee injuries with hemarthrosis. Am J Sports Med 8:9-14

3. Kopf S, Beaufils P, Hirschmann MT, Rotigliano N, Ollivier M, Pereira H et al (2020) Management of traumatic meniscus tears: the 2019 ESSKA meniscus consensus. Knee Surg Sports Traumatol Arthrosc 28:1177-1194

4. Beaufils P, Becker R, Kopf S, Englund M, Verdonk R, Ollivier M et al (2017) Surgical management of degenerative meniscus lesions: the 2016 ESSKA meniscus consensus. Knee Surg Sports Traumatol Arthrosc 25:335-346

5. Englund M, Guermazi A, Gale D, Hunter DJ, Aliabadi P, Clancy M et al (2008) Incidental meniscal findings on knee MRI in middle-aged and elderly persons. N Engl J Med 359:1108-1115 
6. Parker BR, Hurwitz S, Spang J, Creighton R, Kamath G (2016) Surgical trends in the treatment of meniscal tears: analysis of data from the American Board of Orthopaedic Surgery Certification Examination Database. Am J Sports Med 44:1717-1723

7. Espejo-Reina A, Aguilera J, Espejo-Reina MJ, Espejo-Reina MP, Espejo-Baena A (2019) One-third of meniscal tears are repairable: an epidemiological study evaluating meniscal tear patterns in stable and unstable knees. Arthroscopy 35:857-863

8. Fairbank TJ (1948) Knee joint changes after meniscectomy. J Bone Joint Surg Br 30b:664-670

9. Abram SGF, Judge A, Beard DJ, Carr AJ, Price AJ (2019) Long-term rates of knee arthroplasty in a cohort of 834393 patients with a history of arthroscopic partial meniscectomy. Bone Joint J 101-b:1071-1080

10. Paxton ES, Stock MV, Brophy RH (2011) Meniscal repair versus partial meniscectomy: a systematic review comparing reoperation rates and clinical outcomes. Arthroscopy 27:1275-1288

11. Pengas IP, Assiotis A, Nash W, Hatcher J, Banks J, McNicholas MJ (2012) Total meniscectomy in adolescents: a 40-year follow-up. J Bone Joint Surg Br 94:1649-1654

12. Persson F, Turkiewicz A, Bergkvist D, Neuman P, Englund M (2018) The risk of symptomatic knee osteoarthritis after arthroscopic meniscus repair vs partial meniscectomy vs the general population. Osteoarthr Cartil 26:195-201

13. Barnds B, Morris B, Mullen S, Schroeppel JP, Tarakemeh A, Vopat BG (2019) Increased rates of knee arthroplasty and cost of patients with meniscal tears treated with arthroscopic partial meniscectomy versus non-operative management. Knee Surg Sports Traumatol Arthrosc 27:2316-2321

14. Faucett SC, Geisler BP, Chahla J, Krych AJ, Kurzweil PR, Garner AM et al (2019) Meniscus root repair vs Meniscectomy or nonoperative management to prevent knee osteoarthritis after medial meniscus root tears: clinical and economic effectiveness. Am J Sports Med 47:762-769

15. Verdonk R, Madry H, Shabshin N, Dirisamer F, Peretti GM, Pujol N et al (2016) The role of meniscal tissue in joint protection in early osteoarthritis. Knee Surg Sports Traumatol Arthrosc 24:1763-1774

16. Masouros SD, McDermott ID, Amis AA, Bull AMJ (2008) Biomechanics of the meniscus-meniscal ligament construct of the knee. Knee Surg Sports Traumatol Arthrosc 16:1121-1132

17. Jagodzinski M, Müller W, Friederich N (2016) Rotation. In: Jagodzinski M, Friederich N, Müller W (eds) Das Knie, vol 2. Springer, Berlin, Heidelberg, pp 60-89

18. Thompson WO, Thaete FL, Fu FH, Dye SF (1991) Tibial meniscal dynamics using three-dimensional reconstruction of magnetic resonance images. Am J Sports Med 19:210-215 discussion 215-216

19. Novaretti JV, Lian J, Sheean AJ, Chan CK, Wang JH, Cohen M et al (2019) Lateral meniscal allograft transplantation with bone block and suture-only techniques partially restores knee kinematics and forces. Am J Sports Med 47:2427-2436

20. Petersen W, Tillmann B (1998) Collagenous fibril texture of the human knee joint menisci. Anat Embryol (Berl) 197:317-324

21. McDermott ID, Amis AA (2006) The consequences of meniscectomy. J Bone Joint Surg Br 88:1549-1556

22. Baratz ME, Fu FH, Mengato R (1986) Meniscal tears: the effect of meniscectomy and of repair on intraarticular contact areas and stress in the human knee:a preliminary report. Am J Sports Med 14:270-275

23. Perez-Blanca A, Espejo-Baena A, Amat Trujillo D, Prado Nóvoa M, Espejo-Reina A, Quintero López C et al (2016) Comparative biomechanical study on contact alterations after lateral meniscus posterior root avulsion, Transosseous reinsertion, and Total Meniscectomy. Arthroscopy 32:624-633

24. Winkler PW, Wierer G, Csapo R, Hepperger C, Heinzle B, Imhoff AB et al (2020) Quantitative evaluation of dynamic lateral meniscal extrusion after radial tear repair. Orthop J Sports Med 8:2325967120914568

25. Bedi A, Kelly N, Baad M, Fox AJ, Ma Y, Warren RF et al (2012) Dynamic contact mechanics of radial tears of the lateral meniscus: implications for treatment. Arthroscopy 28:372-381

26. Ode GE, Van Thiel GS, McArthur SA, Dishkin-Paset J, Leurgans SE, Shewman EF et al (2012) Effects of serial sectioning and repair of radial tears in the lateral meniscus. Am J Sports Med 40:1863-1870

27. Padalecki JR, Jansson KS, Smith SD, Dornan GJ, Pierce CM, Wijdicks CA et al (2014) Biomechanical consequences of a complete radial tear adjacent to the medial meniscus posterior root attachment site: in situ pull-out repair restores derangement of joint mechanics. Am J Sports Med 42:699-707
28. Diermeier T, Beitzel K, Bachmann L, Petersen W, Esefeld K, Wörtler K et al (2019) Mountain ultramarathon results in temporary meniscus extrusion in healthy athletes. Knee Surg Sports Traumatol Arthrosc 27:2691-2697

29. Karpinski K, Diermeier T, Willinger L, Imhoff AB, Achtnich A, Petersen W (2019) No dynamic extrusion of the medial meniscus in ultrasound examination in patients with confirmed root tear lesion. Knee Surg Sports Traumatol Arthrosc 27:3311-3317

30. Arnoczky SP, Warren RF (1982) Microvasculature of the human meniscus. Am J Sports Med 10:90-95

31. Cooper DE, Arnoczky SP, Warren RF (1990) Arthroscopic meniscal repair. Clin Sports Med 9:589-607

32. Musahl V, Citak M, O'Loughlin PF, Choi D, Bedi A, Pearle AD (2010) The effect of medial versus lateral meniscectomy on the stability of the anterior cruciate ligament-deficient knee. Am J Sports Med 38:1591-1597

33. Novaretti JV, Lian J, Patel NK, Chan CK, Cohen M, Musahl V et al (2020) Partial lateral Meniscectomy affects knee stability even in anterior cruciate ligament-intact knees. J Bone Joint Surg Am 102:567-573

34. Drobnič M, Ercin E, Gamelas J, Papacostas ET, Slynarski K, Zdanowicz U et al (2019) Treatment options for the symptomatic post-meniscectomy knee. Knee Surg Sports Traumatol Arthrosc 27:1817-1824

35. Chatain F, Adeleine P, Chambat P, Neyret P (2003) A comparative study of medial versus lateral arthroscopic partial meniscectomy on stable knees: 10year minimum follow-up. Arthroscopy 19:842-849

36. Hoser C, Fink C, Brown C, Reichkendler M, Hackl W, Bartlett J (2001) Longterm results of arthroscopic partial lateral meniscectomy in knees without associated damage. J Bone Joint Surg Br 83:513-516

37. Mariani PP, Garofalo R, Margheritini F (2008) Chondrolysis after partial lateral meniscectomy in athletes. Knee Surg Sports Traumatol Arthrosc 16:574-580

38. Sonnery-Cottet $\mathrm{B}$, Archbold $\mathrm{P}$, Thaunat M, Carnesecchi O, Tostes M Chambat P (2014) Rapid chondrolysis of the knee after partial lateral meniscectomy in professional athletes. Knee 21:504-508

39. Willinger L, Foehr P, Achtnich A, Forkel P, Voss A, Liska F et al (2019) Effect of lower limb alignment in medial meniscus-deficient knees on Tibiofemoral contact pressure. Orthop J Sports Med 7:2325967118824611

40. Willinger L, Lang JJ, Berthold D, Muench LN, Achtnich A, Forkel P et al (2020) Varus alignment aggravates tibiofemoral contact pressure rise after sequential medial meniscus resection. Knee Surg Sports Traumatol Arthrosc 28:1055-1063

41. Willinger L, Lang JJ, von Deimling C, Diermeier T, Petersen W, Imhoff AB et al (2020) Varus alignment increases medial meniscus extrusion and peak contact pressure: a biomechanical study. Knee Surg Sports Traumatol Arthrosc 28:1092-1098

42. Arner JW, Irvine JN, Zheng L, Gale T, Thorhauer E, Hankins M et al (2016) The effects of anterior cruciate ligament deficiency on the meniscus and articular cartilage: a novel dynamic in vitro pilot study. Orthop J Sports Med 4.2325967116639895

43. McDonald LS, Boorman-Padgett J, Kent R, Stone K, Wickiewicz TL, Pearle AD et al (2016) ACL deficiency increases forces on the medial femoral condyle and the lateral meniscus with applied rotatory loads. J Bone Joint Surg Am 98:1713-1721

44. Brambilla L, Pulici L, Carimati G, Quaglia A, Prospero E, Bait C et al (2015) Prevalence of associated lesions in anterior cruciate ligament reconstruction: correlation with surgical timing and with patient age, sex, and body mass index. Am J Sports Med 43:2966-2973

45. Mehl J, Otto A, Baldino JB, Achtnich A, Akoto R, Imhoff AB et al (2019) The ACL-deficient knee and the prevalence of meniscus and cartilage lesions: a systematic review and meta-analysis (CRD42017076897). Arch Orthop Trauma Surg 139:819-841

46. Goble EM, Kohn D, Verdonk R, Kane SM (1999) Meniscal substitutes--human experience. Scand J Med Sci Sports 9:146-157

47. Johnson LL, Feagin JA Jr (2000) Autogenous tendon graft substitution for absent knee joint meniscus: a pilot study. Arthroscopy 16:191-196

48. Kim JM, Lee BS, Kim KH, Kim KA, Bin SI (2012) Results of meniscus allograft transplantation using bone fixation: 110 cases with objective evaluation. Am J Sports Med 40:1027-1034

49. Koh YG, Kim YS, Kwon OR, Heo DB, Tak DH (2018) Comparative matchedpair analysis of keyhole bone-plug technique versus arthroscopic-assisted pullout suture technique for lateral meniscal allograft transplantation. Arthroscopy 34:1940-1947

50. Marcacci M, Zaffagnini S, Marcheggiani Muccioli GM, Grassi A, Bonanzinga T, Nitri M et al (2012) Meniscal allograft transplantation without bone plugs: a 3-year minimum follow-up study. Am J Sports Med 40:395-403 
51. McCormick F, Harris JD, Abrams GD, Hussey KE, Wilson H, Frank R et al (2014) Survival and reoperation rates after meniscal allograft transplantation: analysis of failures for 172 consecutive transplants at a minimum 2-year follow-up. Am J Sports Med 42:892-897

52. De Coninck T, Elsner JJ, Linder-Ganz E, Cromheecke M, Shemesh M, Huysse W et al (2014) In-vivo evaluation of the kinematic behavior of an artificial medial meniscus implant: a pilot study using open-MRI. Clin Biomech (Bristol, Avon) 29:898-905

53. Vrancken ACT, Hannink G, Madej W, Verdonschot N, van Tienen TG, Buma P (2017) In vivo performance of a novel, anatomically shaped, Total meniscal prosthesis made of polycarbonate urethane: a 12-month evaluation in goats. Am J Sports Med 45:2824-2834

54. Stone KR, Steadman JR, Rodkey WG, Li ST (1997) Regeneration of meniscal cartilage with use of a collagen scaffold. Analysis of preliminary data. J Bone Joint Surg Am 79:1770-1777

55. Verdonk R, Verdonk P, Huysse W, Forsyth R, Heinrichs EL (2011) Tissue ingrowth after implantation of a novel, biodegradable polyurethane scaffold for treatment of partial meniscal lesions. Am J Sports Med 39:774-782

56. Bruns J, Kahrs J, Kampen J, Behrens P, Plitz W (1998) Autologous perichondral tissue for meniscal replacement. J Bone Joint Surg Br 80:918-923

57. Kohn D, Rudert M, Wirth CJ, Plitz W, Reiss G, Maschek H (1997) Medial meniscus replacement by a fat pad autograft. An experimental study in sheep. Int Orthop 21:232-238

58. Kohn D, Wirth CJ, Reiss G, Plitz W, Maschek H, Erhardt W et al (1992) Medial meniscus replacement by a tendon autograft. Experiments in sheep. J Bone Joint Surg Br 74:910-917

59. Rosso F, Bisicchia S, Bonasia DE, Amendola A (2015) Meniscal allograft transplantation: a systematic review. Am J Sports Med 43:998-1007

60. Myers KR, Sgaglione NA, Goodwillie AD (2014) Meniscal scaffolds. J Knee Surg 27:435-442

61. Rodkey WG, DeHaven KE, Montgomery WH 3rd, Baker CL Jr, Beck CL Jr, Hormel SE et al (2008) Comparison of the collagen meniscus implant with partial meniscectomy. A prospective randomized trial. J Bone Joint Surg Am 90:1413-1426

62. Dean CS, Olivetto J, Chahla J, Serra Cruz R, LaPrade RF (2016) Medial meniscal allograft transplantation: the bone plug technique. Arthrosc Tech 5:e329-e335

63. Pollard ME, Kang Q, Berg EE (1995) Radiographic sizing for meniscal transplantation. Arthroscopy 11:684-687

64. Hurley ET, Davey MS, Jamal MS, Manjunath AK, Kingery MT, Alaia MJ et al (2020) High rate of return-to-play following meniscal allograft transplantation. Knee Surg Sports Traumatol Arthrosc. https://doi.org/10. 1007/s00167-020-05956-Z

65. Bin SI, Nha KW, Cheong JY, Shin YS (2018) Midterm and long-term results of medial versus lateral meniscal allograft transplantation: a meta-analysis. Am J Sports Med 46:1243-1250

66. Dienst M, Greis PE, Ellis BJ, Bachus KN, Burks RT (2007) Effect of lateral meniscal allograft sizing on contact mechanics of the lateral tibial plateau: an experimental study in human cadaveric knee joints. Am J Sports Med 35: 34-42

67. Toanen C, Dhollander A, Bulgheroni P, Filardo G, Zaffagnini S, Spalding T et al (2020) Polyurethane meniscal scaffold for the treatment of partial meniscal deficiency: 5-year follow-up outcomes: a European multicentric study. Am J Sports Med. https://doi.org/10.1177/ 0363546520913528363546520913528

68. Schenk L, Bethge L, Hirschmann A, Berbig R, Lüthi U, Arnold MP et al (2020) Ongoing MRI remodeling 3-7 years after collagen meniscus implantation in stable knees. Knee Surg Sports Traumatol Arthrosc 28:1099-1104

69. Monllau JC, Poggioli F, Erquicia J, Ramírez E, Pelfort X, Gelber P et al (2018) Magnetic resonance imaging and functional outcomes after a polyurethane meniscal scaffold implantation: minimum 5-year follow-up. Arthroscopy 34 : 1621-1627

70. Leroy A, Beaufils P, Faivre B, Steltzlen C, Boisrenoult P, Pujol N (2017) Actifit ${ }^{\oplus}$ ) polyurethane meniscal scaffold: MRI and functional outcomes after a minimum follow-up of 5 years. Orthop Traumatol Surg Res 103:609-614

71. Dhollander A, Verdonk P, Verdonk R (2016) Treatment of painful, irreparable partial meniscal defects with a polyurethane scaffold: midterm clinical outcomes and survival analysis. Am J Sports Med 44:2615-2621

72. Filardo G, Kon E, Perdisa F, Sessa A, Di Martino A, Busacca M et al (2017) Polyurethane-based cell-free scaffold for the treatment of painful partial meniscus loss. Knee Surg Sports Traumatol Arthrosc 25:459-467
73. Schuttler KF, Haberhauer F, Gesslein M, Heyse TJ, Figiel J, Lorbach O et al (2016) Midterm follow-up after implantation of a polyurethane meniscal scaffold for segmental medial meniscus loss: maintenance of good clinical and MRI outcome. Knee Surg Sports Traumatol Arthrosc 24:1478-1484

74. Faivre B, Bouyarmane $H$, Lonjon $G$, Boisrenoult P, Pujol N, Beaufils P (2015) Actifit ${ }^{\oplus}$ scaffold implantation: influence of preoperative meniscal extrusion on morphological and clinical outcomes. Orthop Traumatol Surg Res 101:703-708

75. Martín-Hernández C, Ranera-García M, Díaz-Martínez JV, Muniesa-Herrero MP, Floría-Arnal LJ, Osca-Guadalajara M et al (2015) Results of polyurethane implant for persistent knee pain after partial meniscectomy with a minimum of two years follow-up. Rev Esp Cir Ortop Traumatol 59:44-51

76. Schüttler KF, Pöttgen S, Getgood A, Rominger MB, Fuchs-Winkelmann S, Roessler PP et al (2015) Improvement in outcomes after implantation of a novel polyurethane meniscal scaffold for the treatment of medial meniscus deficiency. Knee Surg Sports Traumatol Arthrosc 23:1929-1935

77. Baynat C, Andro C, Vincent JP, Schiele P, Buisson P, Dubrana F et al (2014) Actifit synthetic meniscal substitute: experience with 18 patients in Brest, France. Orthop Traumatol Surg Res 100:S385-S389

78. Gelber PE, Isart A, Erquicia Jl, Pelfort X, Tey-Pons M, Monllau JC (2015) Partial meniscus substitution with a polyurethane scaffold does not improve outcome after an open-wedge high tibial osteotomy. Knee Surg Sports Traumatol Arthrosc 23:334-339

79. Bouyarmane H, Beaufils P, Pujol N, Bellemans J, Roberts S, Spalding T et al (2014) Polyurethane scaffold in lateral meniscus segmental defects: clinical outcomes at 24 months follow-up. Orthop Traumatol Surg Res 100:153-157

80. Kon E, Filardo G, Zaffagnini S, Di Martino A, Di Matteo B, Marcheggiani Muccioli GM et al (2014) Biodegradable polyurethane meniscal scaffold for isolated partial lesions or as combined procedure for knees with multiple comorbidities: clinical results at 2 years. Knee Surg Sports Traumatol Arthrosc 22:128-134

81. De Coninck T, Huysse W, Willemot L, Verdonk R, Verstraete K, Verdonk P (2013) Two-year follow-up study on clinical and radiological outcomes of polyurethane meniscal scaffolds. Am J Sports Med 41:64-72

82. Bulgheroni P, Bulgheroni E, Regazzola G, Mazzola C (2013) Polyurethane scaffold for the treatment of partial meniscal tears. Clinical results with a minimum two-year follow-up. Joints 1:161-166

83. Hirschmann MT, Keller L, Hirschmann A, Schenk L, Berbig R, Luthi U et al (2013) One-year clinical and MR imaging outcome after partial meniscal replacement in stabilized knees using a collagen meniscus implant. Knee Surg Sports Traumatol Arthrosc 21:740-747

84. Zaffagnini S, Marcheggiani Muccioli GM, Bulgheroni P, Bulgheroni E, Grassi A, Bonanzinga T et al (2012) Arthroscopic collagen meniscus implantation for partial lateral meniscal defects: a 2-year minimum follow-up study. Am J Sports Med 40:2281-2288

85. Efe T, Getgood A, Schofer MD, Fuchs-Winkelmann S, Mann D, Paletta JR et al (2012) The safety and short-term efficacy of a novel polyurethane meniscal scaffold for the treatment of segmental medial meniscus deficiency. Knee Surg Sports Traumatol Arthrosc 20:1822-1830

86. Verdonk P, Beaufils P, Bellemans J, Dijan P, Heinrichs EL, Huysse W et al (2012) Successful treatment of painful irreparable partial meniscal defects with a polyurethane scaffold: two-year safety and clinical outcomes. Am J Sports Med 40:844-853

87. Monllau JC, Gelber PE, Abat F, Pelfort X, Abad R, Hinarejos P et al (2011) Outcome after partial medial meniscus substitution with the collagen meniscal implant at a minimum of 10 years' follow-up. Arthroscopy 27:933-943

88. Bulgheroni P, Murena L, Ratti C, Bulgheroni E, Ronga M, Cherubino P (2010) Follow-up of collagen meniscus implant patients: clinical, radiological, and magnetic resonance imaging results at 5 years. Knee 17:224-229

89. Linke RD, Ulmer M, Imhoff AB (2007) Replacement of the meniscus with a collagen implant (CMI). Eur J Trauma Emerg Surg 33:435-440

90. Genovese $E$, Angeretti $M G$, Ronga $M$, Leonardi A, Novario $R$, Callegari $L$ et al (2007) Follow-up of collagen meniscus implants by MRI. Radiol Med 112: 1036-1048

91. Steadman JR, Rodkey WG (2005) Tissue-engineered collagen meniscus implants: 5- to 6-year feasibility study results. Arthroscopy 21:515-525

92. Rodkey WG, Steadman JR, Li ST (1999) A clinical study of collagen meniscus implants to restore the injured meniscus. Clin Orthop Relat Res:S281-S292

93. Zaffagnini S, Marcheggiani Muccioli GM, Lopomo N, Bruni D, Giordano G, Ravazzolo G et al (2011) Prospective long-term outcomes of the medial collagen meniscus implant versus partial medial meniscectomy: a minimum 10-year follow-up study. Am J Sports Med 39:977-985 
94. Filardo G, Andriolo L, Kon E, de Caro F, Marcacci M (2015) Meniscal scaffolds: results and indications. A systematic literature review. Int Orthop 39:35-46

95. Vrancken AC, Eggermont $F$, van Tienen $T G$, Hannink G, Buma $P$, Janssen D et al (2016) Functional biomechanical performance of a novel anatomically shaped polycarbonate urethane total meniscus replacement. Knee Surg Sports Traumatol Arthrosc 24:1485-1494

96. Shemesh M, Shefy-Peleg A, Levy A, Shabshin N, Condello V, Arbel R et al (2020) Effects of a novel medial meniscus implant on the knee compartments: imaging and biomechanical aspects. Biomech Model Mechanobiol. https://doi.org/10.1007/s10237-020-01323-6

97. Active Implants LCC. The NUsurface ${ }^{\circledast}$ Meniscus Implant. URL: https:// activeimplants.com/products/nusurface-meniscus-implant/. Accessed 13.04. 2020, 2020

98. Stärke C, Kopf S, Becker R (2017) Indikation und Grenzen des Meniskusersatzes. Orthopade 46:831-838

99. Langer R, Vacanti JP (1993) Tissue engineering. Science 260:920-926

100. Lyons LP, Hidalgo Perea S, Weinberg JB, Wittstein JR, McNulty AL (2019) Meniscus-Derived matrix bioscaffolds: effects of concentration and crosslinking on meniscus cellular responses and tissue repair. Int J Mol Sci 21(1): 44

101. Ruprecht JC, Waanders TD, Rowland CR, Nishimuta JF, Glass KA, Stencel J et al (2019) Meniscus-derived matrix scaffolds promote the integrative repair of meniscal defects. Sci Rep 9:8719

102. Rothrauff BB, Shimomura K, Gottardi R, Alexander PG, Tuan RS (2017) Anatomical region-dependent enhancement of 3-dimensional chondrogenic differentiation of human mesenchymal stem cells by soluble meniscus extracellular matrix. Acta Biomater 49:140-151

103. Shimomura K, Rothrauff BB, Tuan RS (2017) Region-specific effect of the Decellularized meniscus extracellular matrix on Mesenchymal stem cellbased meniscus tissue engineering. Am J Sports Med 45:604-611

104. Rothrauff BB, Yang G, Tuan RS (2017) Tissue-specific bioactivity of soluble tendon-derived and cartilage-derived extracellular matrices on adult mesenchymal stem cells. Stem Cell Res Ther 8:133

105. Chen M, Feng Z, Guo W, Yang D, Gao S, Li Y et al (2019) PCL-MECM-based hydrogel hybrid scaffolds and meniscal Fibrochondrocytes promote whole meniscus regeneration in a rabbit Meniscectomy model. ACS Appl Mater Interfaces 11:41626-41639

106. Bilgen B, Jayasuriya CT, Owens BD (2018) Current concepts in meniscus tissue engineering and repair. Adv Healthc Mater 7:e1701407

107. Shimomura K, Hamamoto S, Hart DA, Yoshikawa H, Nakamura N (2018) Meniscal repair and regeneration: current strategies and future perspectives. J Clin Orthop Trauma 9:247-253

108. Lee CH, Rodeo SA, Fortier LA, Lu C, Erisken C, Mao JJ (2014) Proteinreleasing polymeric scaffolds induce fibrochondrocytic differentiation of endogenous cells for knee meniscus regeneration in sheep. Sci Transl Med 6:266ra171

109. Nakagawa Y, Fortier LA, Mao JJ, Lee CH, Goodale MB, Koff MF et al (2019) Long-term evaluation of meniscal tissue formation in 3-dimensional-printed scaffolds with sequential release of connective tissue growth factor and TGF-B3 in an ovine model. Am J Sports Med 47:2596-2607

110. Ferris DJ, Frisbie DD, Kisiday JD, Mcllwraith CW, Hague BA, Major MD et al (2014) Clinical outcome after intra-articular administration of bone marrow derived mesenchymal stem cells in 33 horses with stifle injury. Vet Surg 43: 255-265

111. Hatsushika D, Muneta T, Nakamura T, Horie M, Koga $H$, Nakagawa $Y$ et al (2014) Repetitive allogeneic intraarticular injections of synovial mesenchymal stem cells promote meniscus regeneration in a porcine massive meniscus defect model. Osteoarthr Cartil 22:941-950

112. Nakagawa $Y$, Muneta T, Kondo S, Mizuno M, Takakuda K, Ichinose S et al (2015) Synovial mesenchymal stem cells promote healing after meniscal repair in microminipigs. Osteoarthr Cartil 23:1007-1017

113. Chen C, Song J, Qiu J, Zhao J (2020) Repair of a meniscal defect in a rabbit model through use of a Thermosensitive, injectable, in situ Crosslinked hydrogel with encapsulated bone Mesenchymal stromal cells and transforming growth factor $\beta 1$. Am J Sports Med 48:884-894

114. Rothrauff BB, Sasaki H, Kihara S, Overholt KJ, Gottardi R, Lin H et al (2019) Point-of-care procedure for enhancement of meniscal healing in a goat model utilizing Infrapatellar fat pad-derived stromal vascular fraction cells seeded in Photocrosslinkable hydrogel. Am J Sports Med 47:3396-3405
115. Vangsness CT Jr, Farr J 2nd, Boyd J, Dellaero DT, Mills CR, LeRoux-Williams M (2014) Adult human mesenchymal stem cells delivered via intra-articular injection to the knee following partial medial meniscectomy: a randomized, double-blind, controlled study. J Bone Joint Surg Am 96:90-98

116. Jacob G, Shimomura K, Krych AJ, Nakamura N (2019) The meniscus tear: a review of stem cell therapies. Cells 9(1):92

117. Jones IA, Chen X, Evseenko D, Vangsness CT Jr (2019) Nomenclature inconsistency and selective outcome reporting hinder understanding of stem cell therapy for the knee. J Bone Joint Surg Am 101:186-195

118. Chu CR, Rodeo S, Bhutani N, Goodrich LR, Huard J, Irrgang J et al (2019) Optimizing clinical use of biologics in Orthopaedic surgery: consensus recommendations from the 2018 AAOS/NIH U-13 conference. J Am Acad Orthop Surg 27:e50-e63

119. Murray IR, Geeslin AG, Goudie EB, Petrigliano FA, LaPrade RF (2017) Minimum information for studies evaluating biologics in Orthopaedics (MIBO): platelet-rich plasma and Mesenchymal stem cells. J Bone Joint Surg Am 99:809-819

120. O'Keefe RJ, Tuan RS, Lane NE, Awad HA, Barry F, Bunnell BA et al (2020) American Society for Bone and Mineral Research-Orthopaedic Research Society Joint Task Force Report on cell-based therapies. J Bone Miner Res 35:3-17

121. Lamplot JD, Rodeo SA, Brophy RH (2020) A practical guide for the current use of biologic therapies in sports medicine. Am J Sports Med 48:488-503

\section{Publisher's Note}

Springer Nature remains neutral with regard to jurisdictional claims in published maps and institutional affiliations.

\section{Submit your manuscript to a SpringerOpen ${ }^{\circ}$ journal and benefit from:}

- Convenient online submission

- Rigorous peer review

- Open access: articles freely available online

- High visibility within the field

- Retaining the copyright to your article

Submit your next manuscript at $>$ springeropen.com 